\title{
Anxiety, depression, and attachment before and after the first-trimester screening for Down syndrome: comparing couples who undergo ART with those who conceive spontaneously
}

\author{
Laura Udry-Jørgensen ${ }^{1 \dagger}$, Joëlle Darwiche ${ }^{2 * \dagger}$, Marc Germond ${ }^{3}$, Dorothea Wunder ${ }^{4}$ and Yvan Vial ${ }^{4}$ \\ ${ }^{1}$ Institute of Psychotherapy, Department of Psychiatry, Lausanne University Hospital, University of Lausanne, Lausanne, Switzerland \\ ${ }^{2}$ Institute of Psychology, Faculty of Social and Political Sciences, University of Lausanne, Lausanne, Switzerland \\ ${ }^{3}$ FABER Foundation, Lausanne, Switzerland \\ ${ }^{4}$ Department of Gynecology-Obstetrics and Genetics, Lausanne University Hospital, University of Lausanne, Lausanne, Switzerland \\ *Correspondence to: J. Darwiche. E-mail: joelle.darwiche@unil.ch \\ †The authors consider that the first two authors should be regarded as joint first authors
}

\begin{abstract}
Objectives This study's aim was to describe the emotional status of parents to be before and after the first-trimester combined prenatal screening test.

Methods One hundred three couples participated, of which 52 had undergone an in vitro fertilization/intracytoplasmic sperm injection treatment [assisted reproductive technology (ART)] and 51 had conceived spontaneously. Participants completed the state scale of the State-trait Anxiety Inventory, the Edinburgh Depression Scale, and the Maternal and Paternal Antenatal Attachment Questionnaire before the first-trimester combined prenatal screening test at around 12 weeks of gestational age (T1) and just after receiving the results at approximately 14 weeks of gestational age (T2).
\end{abstract}

Results We observed a significant decrease in anxiety and depression symptoms and a significant increase in attachment from $\mathrm{T} 1$ to $\mathrm{T} 2$. Results showed no differences between groups at either time point, which suggests that ART parents are more similar to than different from parents conceiving spontaneously. Furthermore, given the importance of anxiety during pregnancy, a subsample of women with clinical anxiety was identified. They had significantly higher rates of clinical depression and lower attachment.

Conclusions These results indicate that, regardless of whether conception was through ART or spontaneous, clinical anxiety in women over the prenatal testing period is associated with more vulnerability during pregnancy (i.e. clinical depression and less attachment to fetus). (c) 2015 John Wiley \& Sons, Ltd.

\section{INTRODUCTION}

Noninvasive prenatal screening in the first trimester of pregnancy is a standard procedure in most countries. Although screening has obvious medical benefits, studies over the last 10 years have drawn attention to its psychological impact and suggest that it could be an emotional strain on pregnant women and their partners. ${ }^{1-3}$ Anxiety is one of the most studied emotional variables in this context. It has been shown to be highest before the test and, when results are negative, to diminish afterwards. ${ }^{4-7}$ Prepartum depression in connection with prenatal testing has been much less studied but has been shown to follow the same pattern. ${ }^{8}$ These outcomes suggest that the testing itself is stressful and potentially anxiety inducing. ${ }^{6}$ In contrast, studies on prenatal attachment have shown that using an ultrasound for prenatal screening significantly increases the mother's attachment to her fetus. ${ }^{9}$
By providing visual evidence of fetal viability, the test is a strengthening and positive factor. ${ }^{10}$ Confirmation of viability may be particularly important for couples who conceive through assisted reproductive technology (ART), because they have been shown to be more anxious during pregnancy about the well-being and viability of their fetus. ${ }^{11}$

The first-trimester combined prenatal screening test could therefore be considered a moment of psychological activation - both positive and negative - and thus important for understanding parents' psychological status in early pregnancy. However, it has not to our knowledge been investigated in ART populations. Our aim was therefore to assess anxiety and prepartum depression as indicators of parental psychological distress and prenatal attachment as an early indicator of the parent-child bond. To measure change in these variables, we assessed them before and after 
prenatal screening in both an ART and a spontaneous conception (SC) population.

\section{Anxiety}

Anxiety has been described as an emotional side effect of ART. ${ }^{12-14}$ Higher state anxiety scores have been found in ART populations, ${ }^{11,15-17}$ particularly during early pregnancy. ${ }^{18}$ However, most studies indicate no difference in general anxiety between couples undergoing ART treatment and matched control groups. ${ }^{19}$ One study found that, compared with controls, women who underwent ART experienced a significantly larger decrease in state anxiety as the pregnancy progressed, although no difference in general anxiety was observed. $^{20}$ This could indicate that, although mean global scores are similar, the subjective experience is different for ART women, whose distress could be elevated in early pregnancy. Moreover, regardless of the type of conception, high levels of anxiety during pregnancy are of particular interest: Anxiety disorders during pregnancy have been associated with several risk factors, both immediate - such as preterm birth and low birth weight ${ }^{21}$ - and longitudinal impacting child development negatively on various behavioral and neurobehavioral outcomes. ${ }^{22-26}$ Anxiety disorders also predict the risk of depression in the parents to be in the postpartum period. ${ }^{27,28}$

\section{Prepartum depression}

The prevalence of prepartum depression in community samples has been estimated at around $7 \%$ in the first trimester and tends to increase during pregnancy. Depression has been described as one of the most common psychological difficulties associated with the perinatal period. ${ }^{29,30}$ It has been speculated that couples who undergo ART might have a higher prevalence of prepartum depression because the treatment cycles are a psychological stress burden, which could induce depression. ${ }^{31}$ However, recent studies have shown prepartum depression levels not to be significantly different from those observed in a control population. ${ }^{32-34}$

\section{Prenatal attachment}

Prenatal attachment is the bond that develops between the parents to be and the unborn baby. ${ }^{35}$ It has been shown to predict the mother-infant ${ }^{36}$ and father-infant ${ }^{37}$ postnatal relationships, in terms of both interaction and quality of attachment. Studies have shown that prenatal attachment is a developmental factor that increases with gestational age: The more the pregnancy becomes real, the more the parents bond to the imagined baby. ${ }^{32}$ Studies on prenatal attachment in ART populations have produced mixed results: Some found that ART women had higher prenatal attachment compared with controls, ${ }^{38,39}$ while others found no difference. ${ }^{11,32}$

\section{Prenatal testing}

None of the above studies considered the role of prenatal testing. Yet medical staff need to understand couples' emotional reactions to the psychological experience induced by prenatal testing. Screening asks distressing questions, such as whether the child is viable, healthy, and acceptable for the parents. ${ }^{40}$ These questions are very distressing. With ART couples, who have wanted a child longer, it is even more important to understand the psychological experience associated with prenatal testing. These couples could react in ways that are difficult for medical staff to handle. A better understanding would increase their ability to support these couples.

\section{Aims and hypotheses}

The first aim of the study was to understand the changes in the psychological status of the parents to be from before to after the first-trimester prenatal screening test at around 12 weeks of gestational age, by comparing state anxiety, prenatal attachment, and prepartum depression in couples from an SC group with couples who had undergone in vitro fertilization or intracytoplasmic sperm injection, that is, ART. We hypothesized that, provided that the test results were negative, levels of state anxiety and prepartum depression would decrease and fetal attachment would increase over the period from before (T1) to after (T2) testing. We expected the ART group to be more anxious than the SC group at T1, because of their history of infertility, but similar to the SC group at T2, provided that the test results were negative. In line with most previous findings, we did not expect any difference between the groups in attachment or depression at either time point.

Our second aim was to identify a subgroup that was particularly vulnerable because of anxiety, which has been recognized as key to understanding emotional disorders during pregnancy. We selected the individuals whose anxiety score was above the clinical cut-off and hypothesized that their heightened anxiety would be associated with higher levels of prepartum depression and lower prenatal attachment scores at both time points.

\section{METHOD}

\section{Population}

All patients were offered the same first-trimester combined screening test, followed by a diagnostic test (choriocentesis or amniocentesis) when there was a high risk of trisomy 21. Among the initial 109 couples, the drop-out rate was 5.5\% $(N=6)$. A total of 52 couples had undergone an ART treatment, and 51 had conceived spontaneously. At T2, there were seven high risk results to the screening tests (five in the ART group and two in the SC group). All seven were excluded from further analysis, whatever the result of the diagnostic test, leaving 96 couples. Sociodemographic and medical factors are presented in Table 1.

\section{Procedure}

Participants were recruited by a registered research psychologist during the first trimester, once the pregnancy had been confirmed during the routine doctor's appointment. The couples' obstetrician or midwife presented the study to them and gave them an informational letter. The researcher then called the couples who had agreed to be contacted and fully explained the study. The couples completed an informed 
Table 1 Sociodemographic and medical data for the assisted reproductive technology group and the spontaneous conception group

\begin{tabular}{|c|c|c|c|}
\hline & ART $(N=51)$ & Spontaneous conception $(N=52)$ & $t$-test $p$-values \\
\hline \multicolumn{4}{|l|}{ Sociodemographic data } \\
\hline Women's age in years (mean, SD) & $34.82 \pm 3.86$ & $30.21 \pm 4.13$ & $<0.0001$ \\
\hline Men's age in years (mean, SD) & $37.56 \pm 5.03$ & $32.19 \pm 5.07$ & $<0.0001$ \\
\hline Years of cohabitation (mean, SD) & $6.53 \pm 2.80$ & $3.23 \pm 2.53$ & $<0.0001$ \\
\hline Married $(\%, M)$ & $78.50 \%(40)$ & $42.31 \%(22)$ & $<0.0001$ \\
\hline Years of desiring a child (mean, SD) & $4.21 \pm 1.94$ & $0.80 \pm 0.59$ & $<0.0001$ \\
\hline \multicolumn{4}{|l|}{ Couples' socioeconomic class $1 \%, N^{a}$} \\
\hline Upper & $9.80 \%(5)$ & $13.50 \%(7)$ & \\
\hline Upper middle & $45.10 \%(23)$ & $55.70 \%(29)$ & \\
\hline Middle & $23.50 \%(12)$ & $25.00 \%(13)$ & \\
\hline Lower middle and lower & $21.60 \%(11)$ & $5.80 \%(3)$ & \\
\hline \multicolumn{4}{|l|}{ Medical data } \\
\hline Previous pregnancies $1 \%, \mathrm{~N})$ & $31.37 \%(16)$ & $17.31 \%(9)$ & 0.462 \\
\hline Weeks of amenorrhea T1 (mean, SD) & $11.45 \pm 1.27$ & $11.54 \pm 1.18$ & 0.721 \\
\hline Early pregnancy complications $(\%, N)$ & $13.73 \%(7)$ & $3.85 \%(2)$ & 0.063 \\
\hline Years of infertility work-up (mean, SD) & $2.97 \pm 2.20$ & & \\
\hline \multicolumn{4}{|l|}{ Source of infertility $(\%, M)$} \\
\hline Female origin & $25.50 \%(13)$ & & \\
\hline Male origin & $39.20 \%(20)$ & & \\
\hline Mixed origin & $17.60 \%(9)$ & & \\
\hline Undetermined & $17.70 \%(8)$ & & \\
\hline ICSI $\mid \%, M$ & $64.71 \%(33)$ & & \\
\hline
\end{tabular}

ART, assisted reproductive technology; ICSI, intracytoplasmic sperm injection; SD, standard deviation; T1, before testing.

aSocioeconomic class determined based on each couple's highest indicator of socioeconomic position according to Genoud (201 1) $)^{50}$ : upper (e.g. university-educated senior managers), upper middle (e.g. professionals with a university-level or secondary-level education), middle (e.g. vocationally trained employees), and lower middle and lower (e.g. apprenticeship-trained skilled tradespeople or unskilled laborers).

consent form, a brief questionnaire about their situation (e.g. length of relationship, marital status, and desire to have children), and a brief medical anamnesis (e.g. previous pregnancies and early pregnancy complications) before starting the study. The questionnaires assessing anxiety, depression, and attachment were mailed to the couples with instructions to complete them separately, the day before or the day of (but prior to) the first-trimester combined prenatal screening test and then upon receiving the test results (the same or next day).

The study protocol received approval from the ethics commission of the university hospital (protocol 89/10).

\footnotetext{
Measures

Anxiety

State anxiety was assessed using the S-anxiety scale of the State-trait Anxiety Inventory form $Y-1$ (S-STAI), ${ }^{41}$ which is a 20 -item questionnaire mainly evaluating current tension, nervousness, and worry on 5-point Likert scales. Scores range from 20 to 80 , with higher scores indicating greater anxiety. The internal consistency measures were good in the original questionnaire ( $\alpha$ between 0.86 and 0.95 ) and in our sample at $\mathrm{T} 1(\alpha=0.91)$ and T2 $(\alpha=0.92)$.
}

Clinical anxiety. According to the literature, a score of 40 on the S-STAI is the clinical cut-off during pregnancy. ${ }^{42}$ The 18 women who scored above the cut-off on the S-STAI at both $\mathrm{T} 1$ and T2 - ten in the ART group and eight in the SC group made up a 'clinical anxiety group' within our sample. Only two men scored above the cut-off, and both came from the spontaneous pregnancy group; thus, they were not included in our analysis.

\section{Prepartum depression}

We assessed prepartum depression with the Edinburgh Depression Scale (EDS), ${ }^{43}$ which is a validated ten-item self-rating scale. Each item is rated from 0 to 3 on a Likert scale; the maximum score is 30. The EDS assesses depression's most common symptomatology and has been adapted to and validated for the prenatal period. ${ }^{44,45}$ The internal consistency in the initial validation study was strong $(\alpha=0.87)^{43}$ and good in our sample at T1 $(\alpha=0.87)$ and T2 $(\alpha=0.81)$. According to the literature, scores of 15 or more indicate major prenatal depression. ${ }^{46}$

\section{Prenatal attachment}

Parental attachment to the fetus was assessed with the selfreported Maternal Antenatal Attachment Scale ${ }^{47}$ and Paternal Antenatal Attachment Scale. ${ }^{37}$ The Maternal Antenatal 
Attachment Scale has 19 items, and the Paternal Antenatal Attachment Scale 16. Each item is rated from 1 to 5 on a Likert scale $(1=$ absence of feelings for the fetus; $5=$ very strong feelings for the fetus). The internal consistency was strong in the original validation $(\alpha=0.82)^{47}$ and satisfactory in the French validation study for the total score $(\alpha=0.71)^{48}$ but not for the individual factors. We therefore used only the total score, whose internal consistency in our population was good at T1 $(\alpha=0.81)$ and T2 $(\alpha=0.82)$.

\section{Statistical analysis}

All analyses were performed with the IBM Statistical Package for Social Sciences 22. First, the ART and spontaneous pregnancy groups were compared for relevant sociodemographic and medical data in independent $t$-tests (Table 1). None proved significant; they were therefore not included as covariates in the subsequent analyses. Next, we ran a repeated measure analysis of variance (ANOVA) for men and for women to test our second hypothesis comparing the mean anxiety, depression, and attachment scores from T1 to T2 between groups. Time was a within-subject variable, and group was a between-subject variable, allowing the separate effects of time and group and the interaction between them to be examined for each variable.

We then conducted an ANOVA (i.e. with time as a withinsubject variable and group as a between-subject variable) to compare the mean anxiety, depression, and attachment scores of the low-anxiety group with those of the clinical anxiety group. Marginal means that were measured for the ANOVAs are provided in Table 2 .

\section{RESULTS}

Anxiety, depression, and attachment across groups and time Descriptive statistics for the study variables are provided in Table 2. Results of the ANOVA indicated that time had a significant main effect, except on women's depression. Anxiety decreased significantly in both women, $F(1,85)$
$=11.15, p=0.001$, and men, $F(1,81)=6.38, p=0.013$, as did depression in men, $F(1,81)=4.49, p=0.037$. Attachment increased significantly in both women, $F(1,85)=18.27$, $p<0.001$, and men $F(1,81)=14.92, p<0.001$.

There were no significant group effect and no significant interaction effect for men, but there was an interaction effect between time and group for women. Women in the ART group experienced a greater decrease from $\mathrm{T} 1$ to $\mathrm{T} 2$ in anxiety than women in the SC group, whose anxiety decreased only slightly, $F(1,85)=4.62, p=0.034$. For depression, a similar pattern was observed: Prepartum depression tended to decrease in women from the ART group but was stable in women in the SC group, $F(1,85)=3.87, p=0.052$.

\section{Clinical anxiety among women}

Sociodemographically, there was no significant difference between the 18 women who scored above the clinical cut-off for anxiety (clinical anxiety group: $N=10$ ART group and $N=8$ SC group) and those who scored below it (low-anxiety group: $N=78)$ in terms of age $(M=32.26, S D=4.36 ; \quad M=32.29$, $S D=5.14 ; \quad t(23.59)=-0.027, \quad p=0.98), \quad$ socioeconomic class $(M=3.35, S D=0.90 ; M=3.47, S D=1.01 ; t(22.52)=-0.66, p=0.52)$, or years of desiring a child $(M=2.55, S D=2.29 ; \quad M=2.28$, $S D=2.01 ; t(25.03)=0.47, p=0.64)$.

Descriptive statistics for the study variables are provided in Table 3. Time had a main effect on anxiety and attachment in both the clinical anxiety and low-anxiety groups but no significant effect on depression. Anxiety tended to decrease $F(1,85)=3.80, p=0.055$. The increase in attachment was significant $F(1,85)=13.23, p<0.000$.

Furthermore, the group effect was a main effect and significant for all variables at both time points, indicating that women in the clinical anxiety group were significantly more anxious, $F(1,85)=102.73, p<0.000$; more depressed, $F(1,85)=20.39, p<0.000$; and less attached to their fetus, $F(1,85)=102.73, p=0.009$, compared with the low-anxiety group. In addition, four of the women in the clinical anxiety

Table 2 Mean scores of anxiety, depression, and attachment for the assisted reproductive technology group and the spontaneous conception group, before and after the first round of prenatal testing

\begin{tabular}{|c|c|c|c|c|}
\hline & \multicolumn{2}{|r|}{$\mathrm{T} 1$} & \multicolumn{2}{|r|}{$\mathrm{T} 2$} \\
\hline & ARTN= 47 & Spontaneous pregnancy $N=49$ & $\mathrm{ARTN}=47$ & Spontaneous pregnancy $N=49$ \\
\hline & $M(S D)$ & $M(S D)$ & $M(S D)$ & $M(S D)$ \\
\hline \multicolumn{5}{|l|}{ Anxiety } \\
\hline Women & $38.64(11.29)$ & $35.81(9.61)$ & $33.41(9.01)$ & $34.51(11.01)$ \\
\hline Men & $33.92(8.25)$ & $31.48(8.46)$ & $30.06(5.81)$ & $29.59(7.67)$ \\
\hline \multicolumn{5}{|l|}{ Depression } \\
\hline Women & $6.53(6.21)$ & $5.21(5.26)$ & $5.95(4.39)$ & $5.27(4.26)$ \\
\hline Men & $3.44(2.92)$ & $3.15(3.07)$ & $2.74(2.65)$ & $2.72(2.72)$ \\
\hline \multicolumn{5}{|l|}{ Attachment } \\
\hline Women & $73.77(7.06)$ & $73.18(8.86)$ & $76.13(5.56)$ & $75.75(9.04)$ \\
\hline Men & $57.23(6.46)$ & $56.87(7.13)$ & $58.83(6.65)$ & $58.98(7.52)$ \\
\hline
\end{tabular}

ART, assisted reproductive technology; 11 , before testing; T2, after testing 
Table 3 Mean scores of anxiety, depression, and attachment of women with clinical anxiety and low anxiety before and after the first round of prenatal testing

\begin{tabular}{|c|c|c|c|c|}
\hline & \multicolumn{2}{|c|}{$\mathrm{Tl}$} & \multicolumn{2}{|c|}{ T2 } \\
\hline & Clinical anxiety $(N=18)$ & Low anxiety $(N=78)$ & Clinical anxiety $(N=18)$ & Low anxiety $(N=78)$ \\
\hline & $M(S D)$ & $M(S D)$ & $M(S D)$ & $M(S D)$ \\
\hline Anxiety & $49.33(7.44)$ & $33.96(8.86)$ & $48.44(7.79)$ & $30.32(6.74)$ \\
\hline Depression & $9.17(6.92)$ & $4.95(5.21)$ & $9.64(4.65)$ & $3.89(3.32)$ \\
\hline Attachment & $69.22(10.02)$ & $74.33(7.10)$ & $72.11(7.79)$ & $76.81(7.18)$ \\
\hline
\end{tabular}

$\mathrm{T} 1$, before testing; $\mathrm{T} 2$, after testing.

group $(22 \%)$ scored 15 or above on the EDS, indicating major prenatal depression. None of the others scored above 13. There was no interaction effect between time and group.

\section{DISCUSSION}

Regardless of the mode of conception, anxiety, depression, and attachment scores changed significantly from before to after the combined prenatal screening test. Overall, anxiety and depression decreased, and attachment increased. Results on anxiety confirm findings elsewhere: The prenatal test itself may be stressful and heighten anxiety beforehand, whatever the type of pregnancy. Following results indicating a low risk of trisomy 21, anxiety decreases. As anxiety and depression decrease, attachment increases, indicating that these variables are interrelated. Our hypothesis therefore appears to be supported: Low risk results and a normally progressing pregnancy allow for stronger feelings about the fetus and increasing parent-fetus attachment.

The hypothesis that ART couples would have higher pretest anxiety than SC couples was not confirmed. There was no significant difference between the groups for any of the three variables. However, the pattern for anxiety and depression was different for women in the two groups. The steeper decreases in the ART group could suggest that they feel more relieved by a low-risk prenatal screening test than women from the SC group. In the literature, similar differences have been observed, although not related to the prenatal testing. The decrease in state anxiety was significantly greater even though levels of general anxiety were similar, which could indicate ${ }^{20}$ that women who undergo ART experience early pregnancy differently on a psychological level. Possible explanations could be the longer time to conceive, combined with fear and worries in the older ART group of the increased risk of miscarriage and chromosomal aberrations (e.g. trisomy 21) with age.

The clinical anxiety group had significantly higher mean anxiety scores and rates of prenatal depression symptoms and significantly lower prenatal attachment to the fetus. Anxiety and depression symptoms could be negatively impacting prenatal attachment, which has been shown in other studies. ${ }^{9,35,37,49}$

\section{CONCLUSION}

The psychological status of couples in the ART population was similar to that of couples who conceived spontaneously - challenging the idea that ART populations are more vulnerable. However, there were different patterns - particularly for anxiety, which diminished more in women in the ART group. This may be an indication of how important the combined first-trimester prenatal test is for women who have waited longer before conceiving to confirm the healthy progression of the pregnancy and the viability of the fetus. Finally, we observed that, regardless of the mode of conception, clinical anxiety in the first trimester is closely associated with other risk factors - clinical depression and low attachment to the fetus. These results suggest the importance of heightened attention to and clinical follow-up on anxiety disorders in early pregnancy, both in ART populations and in spontaneous pregnancies, particularly around the time of the firsttrimester combined prenatal screening test. For those couples who receive psychological counseling - particularly common in those who undergo ART treatment - our results strongly suggest that such counseling should address their pregnancy experience and any anxiety. For couples who only meet the gynecologist at the screening, questions about potential fears might expose underlying anxiety. Counseling could then be recommended to ward off depression symptoms and prevent potential difficulties in developing an attachment to the fetus.

\section{ACKNOWLEDGEMENTS}

The authors thank Gholamrezaee Mohammad Mehdi for the statistical analyses and Sarah Zimmer for editing the English version of the manuscript.

\section{WHAT'S ALREADY KNOWN ABOUT THIS TOPIC?}

- Prenatal testing is known to be emotionally distressing.

\section{WHAT DOES THIS STUDY ADD?}

- This study adds to our knowledge about how the prenatal screening test at around 12 weeks of gestational age affects parents who conceive via assisted reproductive technology.

- The influence is similar whatever the mode of conception: Afterwards, anxiety and depression decrease, and prenatal attachment increases.

- Clinical anxiety in women during this period is associated with vulnerability to other risk factors, whatever the mode of conception. 


\section{REFERENCES}

1. Api O, Demar HN, Api M, et al. Anxiety scores before and after genetic sonogram. Arch Gynecol Obstet 2009;280(4):553-8.

2. Kowalcek I. Stress and anxiety associated with prenatal diagnosis. Best Pract Res Clin Obstet Gynaecol 2007;21(2):221-8.

3. Jorgensen FS. Attitudes to prenatal screening, diagnosis and research among pregnant women who accept or decline an alpha-fetoprotein test. Prenat Diagn 1995;15(5):419-29.

4. Rados SN, Kosec V, Gall V. The psychological effects of prenatal diagnostic procedures: maternal anxiety before and after invasive and noninvasive procedures. Prenat Diagn 2013;33(12):1194-200.

5. Tercyak KP, Johnson SB, Roberts SF, et al. Psychological response to prenatal genetic counseling and amniocentesis. Patient Educ Couns 2001;43(1):73-84.

6. Kowalcek I, Huber G, Lammers C, et al. Anxiety scores before and after prenatal testing for congenital anomalies. Arch Gynecol Obstet 2003;267 (3):126-9.

7. Green JM, Hewison J, Bekker HL, et al. Psychosocial aspects of genetic screening of pregnant women and newborns: a systematic review. Health Technol Assess 2004;8(33):1-109.

8. Kowalcek I, Muhlhoff A, Bachmann S, et al. Depressive reactions and stress related to prenatal medicine procedures. Ultrasound Obstet Gynecol 2002;19(1):18-23.

9. Yarcheski A, Mahon NE, Yarcheski TJ, et al. A meta-analytic study of predictors of maternal-fetal attachment. Int J Nurs Stud 2009;46 (5):708-15.

10. Sandbrook SP, Adamson-Macedo EN. Maternal-fetal attachment: searching for a new definition. Neuroendocrinol Lett 2004;25: 169-82.

11. McMahon CA, Ungerer JA, Beaurepaire J, et al. Anxiety during pregnancy and fetal attachment after in-vitro fertilization conception. Hum Reprod 1997;12(1):176-82.

12. vanBalen $\mathrm{F}$, Naaktgeboren $\mathrm{N}$, TrimbosKemper TCM. In-vitro fertilization: the experience of treatment, pregnancy and delivery. Hum Reprod 1996;11(1):95-8.

13. Ulrich D, Gagel DE, Hemmerling A, et al. Couples becoming parents: something special after IVF? J Psychosomat Obstet Gynecol 2004;25 (2):99-113.

14. McMahon CA, Tennant C, Ungerer J, et al. 'Don't count your chickens': a comparative study of the experience of pregnancy after IVF conception. J Reprod Infant Psychol 1999;17(4):345-56.

15. Hjelmstedt A, Widstrom AM, Wramsby $\mathrm{H}$, et al. Patterns of emotional responses to pregnancy, experience of pregnancy and attitudes to parenthood among IVF couples: a longitudinal study. J Psychosomat Obstet Gynecol 2003;24(3):153-62.

16. Cohen J, McMahon C, Tennant C, et al. Psychosocial outcomes for fathers after IVF conception: a controlled prospective investigation from pregnancy to four months postpartum. Repr Techn 2001;10:126-31.

17. Glazebrook C, Cox S, Oates M, et al. Psychological adjustment during pregnancy and the postpartum period in a single and multiple in vitro fertilization births: a review and preliminary findings from an ongoing study. Repr Techn 2001;10.

18. Darwiche J, Lawrence C, Vial Y, et al. Anxiety and psychological stress before prenatal screening in first-time mothers who conceived through IVF/ICSI or spontaneously. Women Health 2014;54(5):474-85.

19. Hammarberg K, Fisher JRW, Wynter KH. Psychological and social aspects of pregnancy, childbirth and early parenting after assisted conception: a systematic review. Hum Reprod Update 2008;14 (5):395-414.

20. Klock SC, Greenfeld DA. Psychological status of in vitro fertilization patients during pregnancy: a longitudinal study. Fertil Steril 2000;73 (6):1159-64.

21. Ding XX, Wu YL, Xu SJ, et al. Maternal anxiety during pregnancy and adverse birth outcomes: a systematic review and meta-analysis of prospective cohort studies. J Affect Disord 2014;159:103-10.

22. Beydoun H, Saftlas AF. Physical and mental health outcomes of prenatal maternal stress in human and animal studies: a review of recent evidence. Paediatr Perinat Epidemiol 2008;22(5):438-66.

23. Van den Bergh BRH, Marcoen A. High antenatal maternal anxiety is related to ADHD symptoms, externalizing problems, and anxiety in 8- and 9-year-olds. Child Dev 2004;75(4):1085-97.
24. Van den Bergh BRH, Mulder EJH, Mennes M, et al. Antenatal maternal anxiety and stress and the neurobehavioural development of the fetus and child: links and possible mechanisms. A review. Neurosci Biobehav Rev 2005;29(2): 237-58.

25. McMahon CA, Boivin J, Gibson FL, et al. Pregnancy-specific anxiety, ART conception and infant temperament at 4 months post-partum. Hum Reprod 2013;28(4):997-1005.

26. Field T, Diego M, Hernandez-Reif M, et al. Pregnancy anxiety and comorbid depression and anger: effects on the fetus and neonate. Depress Anxiety 2003;17(3):140-51.

27. Grant KA, McMahon C, Austin MP. Maternal anxiety during the transition to parenthood: a prospective study. J Affect Disord 2008;108 (1-2):101-11.

28. Ohara MW, Swain AM. Rates and risk of postpartum depression - a meta-analysis. Int Rev Psychiatr 1996;8(1):37-54.

29. Bennett HA, Einarson A, Taddio A, et al. Prevalence of depression during pregnancy: systematic review. Obstet Gynecol 2004;103 (4):698-709

30. Gavin NI, Gaynes BN, Lohr KN, et al. Perinatal depression - a systematic review of prevalence and incidence. Obstet Gynecol 2005;106(5):1071-83.

31. Verhaak CM, Smeenk JMJ, Evers AWM, et al. Women's emotional adjustment to IVF: a systematic review of 25 years of research. Hum Reprod Update 2007;13(1):27-36.

32. Hjelmstedt A, Widstrom AM, Collins A. Psychological correlates of prenatal attachment in women who conceived after in vitro fertilization and women who conceived naturally. Birth-Issue Perinat Care 2006;33 (4):303-10.

33. Harf-Kashdaei E, Kaitz M. Antenatal moods regarding self, baby, and spouse among women who conceived by in vitro fertilization. Fertil Steril 2007;87(6):1306-13.

34. Raguz N, McDonald SW, Metcalfe A, et al. Mental health outcomes of mothers who conceived using fertility treatment. Reprod Health 2014;11:7.

35. Condon JT, Corkindale C. The correlates of antenatal attachment in pregnant women. Br J Med Psychol 1997;70(4):359-72.

36. Siddiqui A, Hägglöf B. Does maternal prenatal attachment predict postnatal mother-infant interaction? Early Hum Dev 2000;59 (1):13-25.

37. Condon J, Corkindale C, Boyce P, et al. A longitudinal study of father-toinfant attachment: antecedents and correlates. J Reprod Infant Psychol 2013;31(1):15-30.

38. Chen CJ, Chen YC, Sung HC, et al. Perinatal attachment in naturally pregnant and infertility-treated pregnant women in Taiwan. J Adv Nurs 2011;67(10):2200-8.

39. Fisher JRW, Hammarberg K, Baker GHW. Antenatal mood and fetal attachment after assisted conception. Fertil Steril 2008;89 (5):1103-12.

40. Lippman A Prenatal genetic testing and screening - constructing needs and reinforcing inequities. Am J Law Med 1991;17(1-2):15-50.

41. Bruchon-Schweitzer M, Paulahan I. Manuel de l'inventaire d'Anxiété trait-état (forme Y) [Manual of the State-trait Anxiety Inventory]. Bordeaux, France: Health Psychology Laboratory, University of Bordeaux II; 1990

42. Tendais I, Costa R, Conde A, et al. Screening for depression and anxiety disorders from pregnancy to postpartum with the EPDS and STAI. Span J Psychol 2014;17:9.

43. Cox J, Holden J, Sagovsky I. Detection of postnatal depression. Development of the ten item Edinburgh Postnatal Depression Scale. Br J Psychiatry 1987(150):702-86.

44. Bergink V, Kooistra L, Lambregtse-van den Berg MP, et al. Validation of the Edinburgh Depression Scale during pregnancy. J Psychosom Res 2011;70(4):385-9.

45. Adouard FP, Jacquemain F, Glangeaud N, et al. Validation study of French version of the Edinburgh Post Natal Depression Scale (EPDS) during pregnancy. Infant Ment Health J 2000 21(4-5):251

46. Matthey S, Henshaw C, Elliott S, et al. Variability in use of cut-off scores and formats on the Edinburgh Postnatal Depression Scale implications for clinical and research practice. Arch Wom Ment Health 2006;9(6):309-15. 
47. Condon JT. The assessment of antenatal emotional attachmentdevelopment of a questionnaire instrument. Br J Med Psychol 1993;66:167-83.

48. Denis A, Callahan S, Bouvard M. Examen des propriétés psychométriques de la traduction française de la Maternal Antenatal Attachment Scale (MAAS). L’Encéphale.
49. Vreeswijk C, Maas A, Rijk C, et al. Fathers' experiences during pregnancy: paternal prenatal attachment and representations of the fetus. Psychol Men Masculin 2014;15(2):129-37.

50. Genoud PA. Indice de position socioéconomique (IPSE): un calcul simplifié. Fribourg: Université de Fribourg. Retrieved from www.unifr. ch/ipg/assets/files/DocGenoud/IPSE.pdf, 2011. 